\title{
Cold dust and its relation to molecular gas in the dwarf irregular galaxy NGC 4449
}

\author{
C. Böttner, U. Klein, and A. Heithausen \\ Radioastronomisches Institut der Universität Bonn, Auf dem Hügel 71, 53121 Bonn, Germany \\ Received 5 November 2002 / Accepted 24 June 2003

\begin{abstract}
We present observations of the dwarf irregular galaxy NGC 4449 at $850 \mu \mathrm{m}$ and $450 \mu \mathrm{m}$ obtained with SCUBA at the JCMT. The distribution of the cold dust agrees well with that of the $\mathrm{CO}$ and $\mathrm{H} \alpha$ emission. To explain the integrated mmthrough far-infrared continuum spectrum three dust components are required, with temperatures of $16 \mathrm{~K}, 39 \mathrm{~K}$ and $168 \mathrm{~K}$, respectively. The dust mass is dominated by the cold dust component; we derive a total dust mass of $\sim 3.8 \times 10^{6} M_{\odot}$, and with the local gas-to-dust ratio of $\sim 130$ a total gas mass of $M_{\mathrm{HI}+\mathrm{H}_{2}} \sim 4.9 \times 10^{8} M_{\odot}$. Comparison with the HI mass leads to a total molecular gas mass of $\sim 3.4 \times 10^{8} M_{\odot}$. We derive a conversion factor of the CO line intensity to molecular hydrogen column density $X_{\mathrm{CO}}=N_{\mathrm{H}_{2}} / I_{\mathrm{CO}}$ which is at least 11 times larger than the Galactic value. These values are in accord with the lower metallicity of NGC 4449 .
\end{abstract}

Key words. galaxies: individual: NGC 4449 - galaxies: ISM - galaxies: irregular - ISM: dust, extinction

\section{Introduction}

Carbon monoxide (CO) is commonly used as a tracer for molecular gas because the molecular hydrogen $\left(\mathrm{H}_{2}\right)$, the dominant species, lacks strong emission lines from which the column density could be determined. Because the rotational transitions of $\mathrm{CO}$ are relatively easy to excite, it is possible to use the $\mathrm{CO}$ line intensity integrated over all velocities $\left(I_{\mathrm{CO}}=\right.$ $\int T_{\mathrm{mb}} \mathrm{d} v$ ) or the CO luminosity, $L_{\mathrm{CO}}$, in one of these lines to estimate the column density, $N\left(\mathrm{H}_{2}\right)$, and mass, $M\left(\mathrm{H}_{2}\right)$, of molecular hydrogen, provided one knows the correct conversion. For the Milky Way the conversion factor, $X_{\mathrm{CO}}=N_{\mathrm{H}_{2}} / I_{\mathrm{CO}(1-0)}$, has been found to be between $X_{\mathrm{CO}}=1.6 \times 10^{20} \mathrm{~cm}^{-2}\left(\mathrm{~K} \mathrm{~km} \mathrm{~s}^{-1}\right)^{-1}$ (Hunter et al. 1997) and $X_{\mathrm{CO}}=2.3 \times 10^{20} \mathrm{~cm}^{-2}\left(\mathrm{~K} \mathrm{~km} \mathrm{~s}^{-1}\right)^{-1}$ (Strong et al. 1988). Throughout the paper we will use the latter because it is widely used for comparison in the literature. Early on, the question arose whether one can apply this local value to external galaxies, as the relation may strongly depend on the physical conditions such as the radiation field and the metallicity (Dettmar \& Heithausen 1989; Israel 1997; Barone et al. 2000). Dwarf irregular galaxies provide excellent laboratories to investigate $X_{\mathrm{CO}}$ in environments with lower metal abundances and varying excitation conditions.

An alternative tracer for the molecular gas is the cold dust that one can measure at $\mathrm{mm} / \mathrm{submm}$ wavelengths (Guélin et al. 1993; Dumke et al. 1997). The dust mass can be determined from the emission spectrum with some assumptions on the dust emissivity and composition. Adopting a gas-to-dust ratio the

Send offprint requests to: C. Böttner,

e-mail: cboettne@astro.uni-bonn.de dust mass can be used to estimate the molecular gas mass. This is however not a trivial business because the gas-to-dust ratio is derived locally in the Milky Way and may vary for different conditions. The $\mathrm{CO}$ abundance strongly depends on the amount of dust, because the lower the amount of dust, the lower the shielding of molecules, hence they are easier to dissociate, which in turn will render $\mathrm{CO}$ as an unreliable tracer of the molecular gas.

To study the relation of cold dust and molecular gas in low metallicity environments we have therefore decided to observe NGC 4449 with the Submillimetre Common-User Bolometer Array (SCUBA) at the James Clerk Maxwell Telescope (JCMT) at 850 and $450 \mu \mathrm{m}$. Maps of the cold dust in the millimetre and submillimetre regime for dwarf galaxies are rather rare. Together with the investigation of NCG 1569 (Lisenfeld et al. 2002) the study of NGC 4449 presented here is the second of its kind.

The galaxy NGC 4449 has seen numerous studies over essentially the whole electro-magnetic spectrum (see e.g. Hunter et al. 1999; Hill et al. 1998; Klein et al. 1996). It is a nearby Magellanic irregular dwarf galaxy of high surface brightness at a distance of about 3.7 Mpc (Bajaja et al. 1994). The recent investigation of Karachentsev et al. (2003) yielded a distance of $4.12 \pm 0.50 \mathrm{Mpc}$, which agrees with the older value within the errors. Located in the constellation Canes Venatici it is a member of the CVnI group (Sandage 1975), which contains mainly spirals and irregular galaxies. The observed morphology and kinematics of the HI gas suggests a tidal interaction with the nearby dwarf galaxy DDO 125 (Theis \& Kohle 2001) and may explain the high star-formation rate and the huge 
HI streamers surrounding NGC 4449. The metallicity was found to be $12+\log (\mathrm{O} / \mathrm{H})=8.3$ (Lequeux et al. 1979).

Hunter et al. (1986) stated that the spectral distribution of the far-infrared emission from NGC 4449 as observed with IRAS is not different from normal spirals. The ratio of the IRAS flux densities at 100 and $60 \mu \mathrm{m} S_{100 \mu \mathrm{m}} / S_{60 \mu \mathrm{m}} \approx 2$ is at the lower end of the range covered by spirals and indicates a high star formation rate. However, the dust mass calculated from this measurement only relies on the warm dust components, thus representing a lower limit. Our new observations allow to determine the amount of cold dust in NGC 4449 and to study the gas-to-dust ratio in more detail. In Sect. 2 we describe the observations and data reduction procedure. Section 3 presents the results, while in Sect. 4 a comparison with the gas in NGC 4449 is made. A summary is given in Sect. 5.

\section{Observations and data reduction}

NGC 4449 was imaged simultaneously at 850 and $450 \mu \mathrm{m}$ using the SCUBA camera at the JCMT in January 18, 1999. The observations were made in the jiggle mode with a chopper throw of $120^{\prime \prime}$. Two fields of view of about 2.3 with sufficient overlap were observed. The extent of the galaxy at optical wavelengths is about $2^{\prime}$ by $3^{\prime}$. Since we observed about 9 hours with the source rotating on the sky, there may have been some emission from the galaxy itself in the reference beam occasionally. However, by combining all coverages this error should be small compared to the calibration uncertainties. Thus only a diffuse extended dust component possibly associated with the large HI halo would have been missed by our observations.

The total net observing time was 8960 s for field A and $6528 \mathrm{~s}$ for field B. The two fields were placed around a central position at $\alpha_{1950}=12^{\mathrm{h}} 25^{\mathrm{m}} 46^{\mathrm{s}}, \delta_{1950}=44^{\circ} 22^{\prime} 45^{\prime \prime}$ such as to cover the main (CO emitting) body of the galaxy. The whole observing run ( 9 hours in total) had good and stable weather with an optical depth of $\tau_{850} \approx 0.23$ and a seeing of $\sim 0$.' 4 . Flux calibration was made using Mars, which was observed in the middle and at the end of the observing run.

The data have been reduced in the standard manner using the STARLINK reduction package SURF (Jenness \& Lightfoot 1999). Flatfielding was performed to correct for differences in the sensitivity of the bolometers. The atmospheric opacity was determined from skydip observations. Noisy bolometers and spikes were identified and blanked out. Typical pointing corrections were $1^{\prime \prime}$ to $2^{\prime \prime}$, hence small compared to the beam size. From the planet profile, the beam size (HPBW) was estimated to $15^{\prime \prime} .3$ and $8 . .5$ at 850 and $450 \mu \mathrm{m}$, respectively. Figure 1 shows the resulting maps of NGC 4449 at 850 and $450 \mu \mathrm{m}$ in the top panels. The noise levels of the maps are $\sigma=6 \mathrm{mJy} /$ beam at $850 \mu \mathrm{m}$ and $\sigma=18 \mathrm{mJy} /$ beam at $450 \mu \mathrm{m}$.

Observations at $1200 \mu \mathrm{m}$ were previously carried out with the IRAM 30 m telescope on Pico Veleta (Spain) in December 1998, using the 19-channel bolometer array of the Max-PlanckInstitut für Radioastronomie (Kohle 1999). We obtained OnThe-Fly (OTF) maps with sizes between $300^{\prime \prime} \times 200^{\prime \prime}$ and $400^{\prime \prime} \times 300^{\prime \prime}$ in azimuth and elevation, with the telescope moving in azimuth at a speed of $6^{\prime \prime} / \mathrm{s}$. Successive horizontal scans were spaced by $4^{\prime \prime}$ in elevation. The wobbler throw was $45^{\prime \prime}$ and wobbled at $0.5 \mathrm{~Hz}$ in azimuth. During the run the weather conditions were good and the atmosphere stable, with sky opacities between 0.1 and 0.3 at $230 \mathrm{GHz}$. Two channels had to be discarded. The total integration time was about 8 hours. Calibration was made using Mars, which was observed twice during the run. The derived beam-size is 10 .' 8 and the resulting map is shown on the left of the centre row in Fig. 1, smoothed to match the $15^{\prime \prime} .3$ of the $850 \mu \mathrm{m}$ map and to improve the signalto-noise. The noise level of this map is $\sigma=2 \mathrm{mJy} / \mathrm{beam}$.

We have to mention that restoring maps from chopped onthe-fly measurements may cause photometric imperfections, as the information on the large-scale structure (larger than the wobbler throw) is lost. The same holds true of course for the IRAM data: we may have missed a diffuse, extended dust component and hence a noticeable fraction of the integrated flux. Such a component was suggested by Thronson et al. (1987) which detected emission at $150 \mu \mathrm{m}$ with the Kuiper Airborne Observatory (KAO) on scales of up to 4 arcmin at a low signalto-noise ratio. However, this will not change our general conclusions. In particular, if we would be able to correct for a missing large-scale component, we would flatten the slope of the submm spectrum, and increase the dust mass (see Sect. 3).

\section{Results}

Figure 1 exhibits the dust continuum emission of NGC 4449 resulting from our observations, along with images in the $\mathrm{HI}, \mathrm{H} \alpha$ and CO line. The galaxy is clearly detected at all $\mathrm{mm} / \mathrm{submm}$ wavelengths. The morphology of the galaxy is rather similar for all components, except for the neutral atomic hydrogen. The strongest dust emission comes from a bar-like structure prominent in the optical regime, although the brightest regions do not coincide. The optically bright northern region is conspicuous in all maps and manifests a large star-forming complex which is most pronounced in the $\mathrm{H} \alpha$ map. A more detailed comparison is hardly possible, due to the low signal-to-noise and therefore the uncertainties in the exact structures.

By integrating over the whole SCUBA maps we have determined total flux densities to be $S_{850 \mu \mathrm{m}}=1.8 \pm 0.2 \mathrm{Jy}$ and $S_{450 \mu \mathrm{m}}=16.5 \pm 4.1 \mathrm{Jy}$. No color correction has been made. Because the $\mathrm{CO}(3-2)$-line emission falls into the bolometer bandpass at $850 \mu \mathrm{m}$, we corrected the total continuum flux for the line contribution. We estimated the contamination using the $\mathrm{CO}(1-0)$ map and assuming a line ratio of $I_{\mathrm{CO}(3-2)} / I_{\mathrm{CO}(1-0)} \approx 0.7$ (Fritz 2002, private communication). We thus obtained a contribution of the $\mathrm{CO}$ line to the total emission of $\sim 12 \%$, resulting in a pure continuum flux of $1.5 \pm 0.3 \mathrm{Jy}$. The contamination at $450 \mu \mathrm{m}$ is negligible compared to the strong thermal dust emission, hence no correction was made. The integrated flux at $1.2 \mathrm{~mm}$ was taken from Kohle (1999) and is also corrected for contributions of the $\mathrm{CO}(2-1)$ line. The quoted errors are formal errors based on the map noise and on the uncertainty of integrating the flux, including calibration errors.

In the literature there are 5 published FIR flux densities for NGC 4449. These are the four IRAS data points between $12 \mu \mathrm{m}$ an $100 \mu \mathrm{m}$ (Hunter et al. 1986), and a $150 \mu \mathrm{m}$ value from KAO observations (Thronson et al. 1987). Together with 

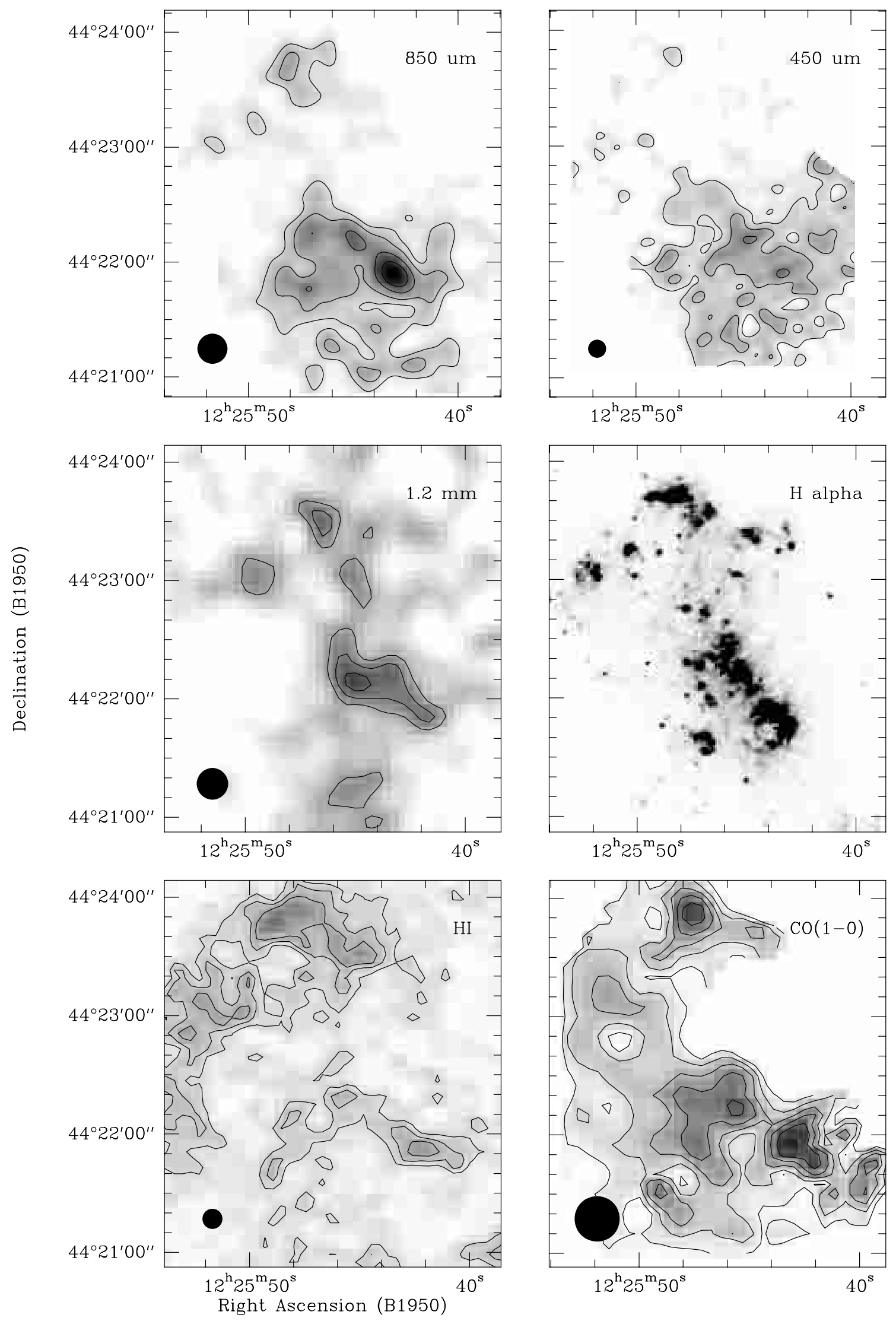

Fig. 1. Intensity maps of NGC 4449 at 850 (upper left) and $450 \mu \mathrm{m}$ (upper right), presented in this work, with contour levels of 2, 3, 4 and $5 \sigma$, where $\sigma$ is the noise level of the map $(\sigma(450 \mu \mathrm{m})=18 \mathrm{mJy} / \mathrm{beam}$ and $\sigma(850 \mu \mathrm{m})=6 \mathrm{mJy} / \mathrm{beam})$; at $1.2 \mathrm{~mm}$ (centre left, from Kohle 1999$)$, with contour levels of 2, 2.5 and $3 \sigma(\sigma(1200 \mu \mathrm{m})=2 \mathrm{mJy} / \mathrm{beam})$; an $\mathrm{H} \alpha$ image (centre right, from Bomans et al. 1997), the integrated $\mathrm{HI}$ (bottom left, from Hunter 1999), with contour levels of 0.06, 0.08 and $0.1 \mathrm{Jy} /$ beam and the integrated $\mathrm{CO}(1-0)$ (bottom right, from Kohle et al. 1998, 1999), with contour levels from 0.5 to $2.5 \mathrm{~K} \mathrm{~km} \mathrm{~s}^{-1}$ in steps of $0.5 \mathrm{~K} \mathrm{~km} \mathrm{~s}^{-1}$. All maps show the same area as that mapped with SCUBA. 
Table 1. IR to submm integrated flux densities of NGC 4449 from $12 \mu \mathrm{m}$ to $1200 \mu \mathrm{m}$. The values at 850 and $1200 \mu \mathrm{m}$ have been corrected for contamination by $\mathrm{CO}$ lines.

\begin{tabular}{rcc}
\hline \hline$\lambda$ & $\begin{array}{c}\text { Flux density } \\
(\mu \mathrm{m})\end{array}$ & Ref. \\
\hline 12 & $2.1 \pm 0.2$ & Hunter et al. (1986) \\
25 & $4.7 \pm 0.5$ & Hunter et al. (1986) \\
60 & $36 \pm 3$ & Hunter et al. (1986) \\
100 & $73 \pm 8$ & Hunter et al. (1986) \\
150 & $100 \pm 20$ & Thronson et al. (1987) \\
450 & $16.5 \pm 4.1$ & this work \\
850 & $1.5 \pm 0.3^{*}$ & this work \\
1200 & $0.26 \pm 0.04^{*}$ & Kohle (1999) \\
\hline
\end{tabular}

* Corrected for CO line contamination.

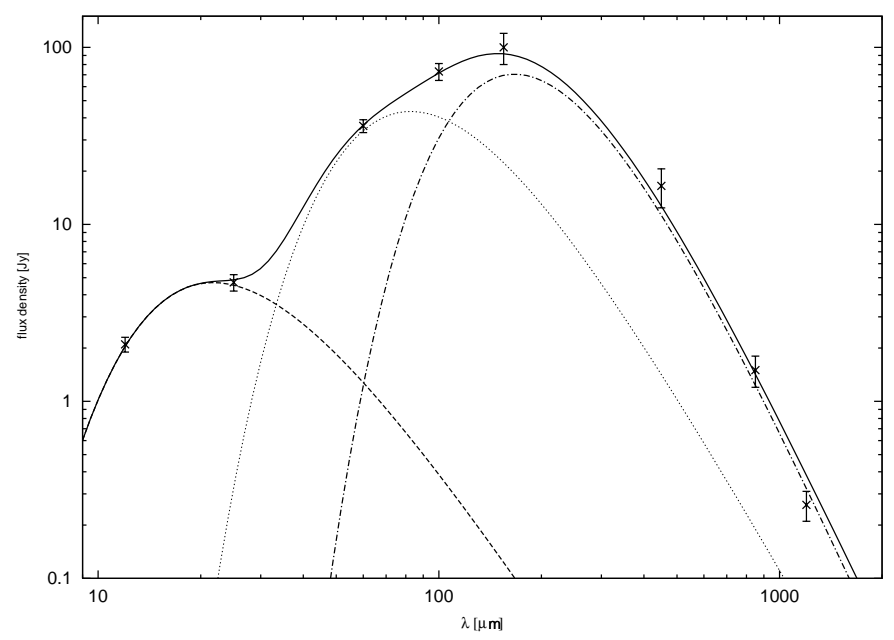

Fig. 2. The mid-infrared to millimeter spectrum of NGC 4449, fitted with three-temperature dust components. Crosses mark flux densities from Table 1. The three dust components have temperatures of $168 \mathrm{~K}$ (dashed line), $39 \mathrm{~K}$ (dotted line) and $16 \mathrm{~K}$ (dashed-dotted line).

the data points presented in this paper this leads to a fair sampling of the spectral energy distribution of NGC 4449 from mm through FIR wavelengths. In Table 1 we have compiled all values, Fig. 2 showing the resulting spectrum.

If we assume the dust grains to be in equilibrium with the radiation field we can describe their emission by a modified black-body law. This will definitively be the case for large grains, which contribute most to the sub-millimetric continuum. They also account for more than $90 \%$ of the dust mass (see e.g. Désert et al. 1990). The emission from 20 to $80 \mu \mathrm{m}$ can originate from very small grains at a significant amount. In this case, with our simple approach we may overestimate the mass of that component by a few percent. However, as we will see below, the mass of this hot component is of almost no importance.

In case of a low optical depth the intensity is given by $F_{\lambda}=B_{\lambda}(T) \cdot k(\lambda)$, where $B_{\lambda}(T)$ is the Planck function, $T$ the dust temperature and $k(\lambda)$ is the absorption coefficient. The wavelength dependence of the latter, which is usually assumed to follow a power-law $k(\lambda) \propto \lambda^{-\beta}$ (see e.g. Alton et al. 2000), is not precisely known. Models of the interstellar dust yield a spectral index $\beta=1 \ldots 2$ for mixtures of small and big grains (e.g. Draine \& Lee 1984). Gordon (1988) has noted a temperature dependence of $\beta$, for the range $1 \ldots 2.5$, and Dunne \& Eales (2001) have derived $\beta \simeq 2$ on a statistical basis for a sample of bright IRAS galaxies.

In order to further analyze the dust components of NGC 4449 we have modeled the spectrum with three dust components at different temperatures. This yields a reasonable fit to the emission spectrum over the entire observed wavelength range. As we have only eight observed spectral data points, we have to limit the model to three components. In reality, the dust is probably at a range of temperatures, reflecting the range of colours and intensities of the heating radiation fields. We decided to use $\beta=1$ for the hot and $\beta=2$ for the warm component, leaving 7 free parameters for the fit. It was not possible to fit the steep slope of the spectrum satisfactorily with $\beta=2$ for the cold component, thus we decided to leave it free up to 2.5, resulting in a value of $\beta=2.3$. Figure 2 shows the result with hot $(T=168 \pm 10 \mathrm{~K}, \beta=1)$, warm $(T=39 \pm 3 \mathrm{~K}, \beta=2)$ and cold $(T=16 \pm 2 \mathrm{~K}, \beta=2.3)$ dust components.

The derived parameters are in accord with other investigations (e.g. Dumke et al. 1997), and agree well with measurements of the diffuse ISM in the Galaxy ( $T \approx 17 \mathrm{~K}, \beta=2)$ by COBE/FIRAS (Reach et al. 1995; Sodroski et al. 1997). In order to properly fit the relatively strong (sub)millimeter emission, a cold component is required. Moreover, because of the low dust emissivity at this temperature, a very large fraction of the total dust has to be cold in order to explain the emission at $850 \mu \mathrm{m}$ and $1200 \mu \mathrm{m}$. An attempt to fit a two-temperature model yields significantly worse results.

From the three-component fit to the spectrum of NGC 4449 we can estimate the dust mass. This can be achieved with a simple equation adapted from Hildebrand (1983), where

$M_{\mathrm{d}}=\frac{D^{2} S_{850 \mu \mathrm{m}}}{B_{850 \mu \mathrm{m}}(T) k_{850 \mu \mathrm{m}}}$.

Here, $B_{850 \mu \mathrm{m}}(T)$ is the Planck function at $850 \mu \mathrm{m}, D$ is the distance and $S_{850 \mu \mathrm{m}}$ the observed flux density. To derive a consistent value of the dust absorption coefficient $k_{850 \mu \mathrm{m}}$ we used the above mentioned power-law $k(\lambda) \propto \lambda^{-\beta}$ and the canonical value of $k_{250 \mu \mathrm{m}}=10 \mathrm{~cm}^{2} \mathrm{~g}^{-1}$ from Hildebrand (1983), extrapolating to the relevant wavelengths and using the associated value of $\beta$. We thus obtained the following values of the dust absorption coefficient for the three different components: $k_{850 \mu \mathrm{m}}=0.6 \mathrm{~cm}^{2} \mathrm{~g}^{-1}$ for the cold $(16 \mathrm{~K})$ component at $850 \mu \mathrm{m}, k_{100 \mu \mathrm{m}}=63 \mathrm{~cm}^{2} \mathrm{~g}^{-1}$ for the warm $(39 \mathrm{~K})$ and $k_{100 \mu \mathrm{m}}=25 \mathrm{~cm}^{2} \mathrm{~g}^{-1}$ for the hot component at $100 \mu \mathrm{m}$. These values are consistent given our assumptions, and especially for the spectral indices used here. However, in the literature one can find a wide range of values for the emissivity and the dependences are only poorly known. As the most extreme cases we quote two values, namely $k_{850 \mu \mathrm{m}}=0.26 \mathrm{~cm}^{2} \mathrm{~g}^{-1}$ (Alton et al. 2002) and $k_{850 \mu \mathrm{m}}=2.4 \mathrm{~cm}^{2} \mathrm{~g}^{-1}$ (Alton et al. 2000).

The three components shown in Fig. 2 result in a very small mass of hot dust, $M_{\mathrm{d}, \mathrm{h}} \simeq 18 M_{\odot}$, a larger mass of warm 
dust, $M_{\mathrm{d}, \mathrm{w}}=0.3 \times 10^{5} M_{\odot}$ and a large mass of the cold dust, $M_{\mathrm{d}, \mathrm{c}}=3.8 \times 10^{6} M_{\odot}$. The total dust mass, $M_{\mathrm{d}}=3.8 \times 10^{6} M_{\odot}$, is thus completely dominated by the cold dust component. The uncertainty of this mass is naturally large (within a factor of 3), and is mainly governed by the peak and the slope of the spectrum between 100-1200 $\mu \mathrm{m}$, and by the assumed dust absorption coefficient, which is not well known. The error in calculating the dust mass caused by using modified Plankians is negligible in view of the large uncertainties in the general grain properties.

Thronson et al. (1987) calculated a somewhat lower mass of $M_{\mathrm{d}} \approx 1.5 \times 10^{6} M_{\odot}$, using their $150 \mu \mathrm{m}$ value and assuming a spectral index of $\beta=1$; the deviation to our value is most likely caused by the then unknown slope of the spectrum in the millimetre regime.

Lisenfeld et al. (2002) have undertaken a similar study of NGC 1569, a galaxy which is presently in the aftermath of a massive burst of star formation. The dust spectrum of that galaxy shows a completely different slope in the range between $150-1200 \mu \mathrm{m}$. The IRAS-flux densities of NGC 1569 indicate a different location of the maximum between 100 and $60 \mu \mathrm{m}$, compared to NGC 4449, indicating the presence of much more hot dust in the former. A very cold component of around $7 \mathrm{~K}$ was needed to properly fit the spectrum, which resulted in a tremendous mass of this very cold dust. The authors stated that such a large amount of cold dust is very unlikely in a dwarf galaxy dominated by intense radiation fields ( 2 orders of magnitude higher than in the solar neighbourhood). Hence, Lisenfeld et al. favour the model of Désert et al. (1990), where the very small grains have optical properties consistent with beta $=1$. Since NGC 1569 has a shallow slope, the resulting fit requires a large contribution of grains emitting like beta $=1$, thus a large contribution of VSG with the Désert's et al. properties. The dust mass derived from this model is much less than that from a fit with modified Plankians and results in a overall gas-to-dust ratio of $M_{\mathrm{g}} / M_{\mathrm{d}} \approx 1500 \ldots 2900$. We have to point out, however, that the physical reason for the steeper slope of the submm dust continuum spectrum of NGC 4449 ( $\beta=2.3 \mathrm{vs}$. $\beta=1.0$ in case of NGC 1569) is not at all obvious.

\section{Comparison with the gas}

The derived dust distribution can be compared with other tracers of the ISM, viz. CO and HI. We also have a $\mathrm{CO}(1-0)$ map of NGC 4449 at our disposal, obtained with the IRAM 30-m telescope on Pico Veleta (Kohle 1998, 1999). Figure 1 shows the integrated map, the angular resolution is $22^{\prime \prime}$. The correlation of $\mathrm{CO}$ and dust is clearly seen. However, the $\mathrm{CO}$ coverage of the galaxy is not uniform, especially in the outer parts so that not all of the structures seen there are reliable.

We have also compared the $\mathrm{H} \alpha$ map published by Bomans et al. (1997) and shown in Fig. 1 to the maps of the dust and the molecular gas. It is evident that the strongest dust emission follows the distribution of intense star formation (as expected). However, this is not a simple one-to-one relation. The same is true for the $\mathrm{CO}$ emission and may indicate dissociation processes as observed in massive star forming regions. There is also significant dust emission away from the star-forming regions, but this is accompanied by $\mathrm{CO}$ radiation and may show a more diffuse component (smaller clouds) of the molecular gas.

A comparison with the HI (Hunter et al. 1999) shows only little correlation. The inner HI of NGC 4449 is organized in a ring-like feature around the optical body. In the northeast one can see part of this ring, whereas in the south there is nearly no $\mathrm{HI}$ within our mapsize. In the center of NGC 4449 only little HI is found, which is expected. The gas should be ionized there, in molecular form or frozen out onto the dust grains.

With the dust mass of $M_{\mathrm{d}}=3.8 \times 10^{6} M_{\odot}$ derived above we can estimate the total gas mass. Using the normal gas-to-dust ratio of $M_{\mathrm{g}} / M_{\mathrm{d}} \approx 130$ (Devereux \& Young 1990) this yields a total gas mass of $M_{\mathrm{HI}+\mathrm{H}_{2}}=4.9 \times 10^{8} M_{\odot}$ for the inner (optical) part of the galaxy. Computing the HI mass within our mapsize yields $M_{\mathrm{HI}}=1.5 \times 10^{8} M_{\odot}$. Subtraction from the total gas mass estimated above results in a mass of molecular hydrogen of $M_{\mathrm{H}_{2}}=3.4 \times 10^{8} M_{\odot}$. From the integrated CO spectrum we derive a CO luminosity of $L_{\mathrm{CO}}=7.9 \times 10^{6} \mathrm{~K} \mathrm{~km} \mathrm{~s}^{-1} \mathrm{pc}^{2}$, which leads to a conversion factor from $\mathrm{CO}$ intensity to $\mathrm{H}_{2}$ column density $X_{\mathrm{CO}}=25 \times 10^{20} \mathrm{~cm}^{-2}\left(\mathrm{~K} \mathrm{~km} \mathrm{~s}^{-1}\right)^{-1}$, i.e. about 11 times the standard Galactic value quoted in Sect. 1. Such an enhanced value is explicable in view of the lower metallicity, of NGC 4449, viz. $12+\log (\mathrm{O} / \mathrm{H})=8.3$ (Lequeux et al. 1979).

Kohle (1999) derived an even higher $X_{\mathrm{CO}}$, assuming virialized cloud complexes in the CO map. He obtained $X_{\mathrm{CO}}=(37 \pm 11) \times 10^{20} \mathrm{~cm}^{-2}\left(\mathrm{~K} \mathrm{~km} \mathrm{~s}^{-1}\right)^{-1}$, which is about 16 times the Galactic value and may represent an upper limit. This would lead to a much higher molecular gas mass and hence to an even higher gas-to-dust ratio. Both scenarios are in agreement with what has been found for other low-mass dwarf irregular galaxies (see e.g. Barone et al. 2000 and references therein). Thronson et al. (1987) derived $X_{\mathrm{CO}} / X_{\mathrm{MW}} \approx 10$ from their KAO data. Based on a much poorer sampling of the dust spectrum using IRAS CPC data Israel (1997) calculated a value of $X_{\mathrm{CO}}=8 \times 10^{20} \mathrm{~cm}^{-2}\left(\mathrm{~K} \mathrm{~km} \mathrm{~s}^{-1}\right)^{-1}$, which is significantly lower than the other values discussed here.

\section{Conclusions}

We have presented new maps of the dwarf galaxy NGC 4449 at 450 and $850 \mu \mathrm{m}$, taken with SCUBA at the JCMT. The integrated flux densities were compared to those obtained with IRAS and KAO. The derived flux densities can be readily explained by the presence of a significant amount of cold dust. We were able to fit the observed SED well with modified Plankians of three dust components, requiring most of the dust to be at a temperature of $16 \mathrm{~K}$, with a spectral index $\beta=2.3$, which is a bit higher than the frequently used value of 2 .

Comparison with $\mathrm{CO}$ observations shows a good coincidence of the cold dust and the molecular gas. The $\mathrm{H} \alpha$ image shows that star-forming regions are related to the dust emission peaks. The main emission comes from the bar-like structure, but there is also weak dust emission away from the main body of NGC 4449, and there may be some more following the $\mathrm{HI}$ ring-structure. 
Assuming a standard local gas-to-dust ratio, we derive a conversion factor $X_{\mathrm{CO}} \approx 25 \times 10^{20} \mathrm{~cm}^{-2}\left(\mathrm{~K} \mathrm{~km} \mathrm{~s}^{-1}\right)^{-1}$, which is 11 times the Galactic value. Given the low metallicity of NGC 4449 (1/3 solar), this is probably still a lower limit, because a standard gas-to-dust ratio may not be applicable. The standard Galactic conversion factor thus strongly underestimates the molecular gas mass in such galaxies, because our tracer - the CO molecule - is less abundant, due to the lower metallicity and/or dissociation. In view of the low metallicity of NGC 4449 this seems in perfect agreement with studies of other dwarf irregulars.

Acknowledgements. We are grateful to Gerald Moriarty-Schieven for performing the observations and to D. A. Hunter for making the $\mathrm{HI}$ data available to us. We are also indebted to C. Kramer and the anonymous referee for many helpful suggestions.

\section{References}

Alton, P. B., Xilouris, E. M., Bianchi, S., et al. 2000, A\&A, 356, 795 Alton, P. B., Bianchi, S., Richter, J., et al. 2002, A\&A, 388, 446

Bajaja, E., Huchtmeier, W. K., \& Klein, U. 1994, A\&A, 285, 385

Barone, L. T., Heithausen, A., Hüttemeister, S., et al. 2000, MNRAS, 317,649

Bomans, D. J., Chu, Y., \& Hopp, U. 1997, AJ, 113, 1678

Désert, F.-X., Boulanger, F., \& Puget, J. L. 1990, A\&A, 237, 215

Dettmar, R. J., \& Heithausen, A. 1989, ApJ, 344, L61

Devereux, N. A., \& Young, J. S. 1990, ApJ, 359, 42

Draine, B. T., \& Lee, H. M. 1984, ApJ, 285, 89

Dumke, M., Braine, J., Krause, M., et al. 1997, A\&A, 325, 124
Dunne, L., \& Eales, S. A. 2001, MNRAS, 327, 697

Gordon, M. A. 1988, A\&A, 331, 509

Guélin, M., Zylka, R., Mezger, P. G., et al. 1993, A\&A, 279, L37

Hildebrand, R. H. 1983, QJRAS, 24, 267

Hill, R. S., Fanelli, M. N., Smith, D. A., et al. 1998, ApJ, 507, 179

Hunter, D. A., Gillett, F. C., Gallagher, J. S., et al. 1986, ApJ, 303, 171

Hunter, D. A., van Woerden, H., \& Gallagher, J. S. 1999, AJ, 118, 2184

Hunter, S. D., et al. 1997, ApJ, 481, 205

Israel, F. P. 1997, A\&A, 328, 471

Jenness, T., \& Lightfoot, J. F. 1998, SURF - SCUBA User Reduction Facility, Starlink User Note 216, Starlink Project, CLRC

Karachentsev, I. D., Sharina, M. E., Dolphin, A. E., et al. 2003, A\&A, 398,467

Klein, U., Hummel, E., Bomans, D. J., \& Hopp, U. 1996, A\&A, 313, 396

Kohle, S., Klein, U., Henkel, C., \& Hunter, D. A. 1998, in The Magellanic Clouds and Other Dwarf Galaxies, ed. T. Richtler, \& J. M. Braun (Shaker Verlag), 265

Kohle, S. 1999, Ph.D. Thesis, Univ. Bonn

Lequeux, J., Peimbert, M., Rayo, J. F., et al. 1979, A\&A, 80, 155

Lisenfeld, U., Israel, F. P., Stil, J. M., \& Sievers, A. 2002, A\&A, 382, 860

Reach, W. T., Dwek, E., Fixsen, D. J., et al. 1995, ApJ, 451, 188

Sandage, A., Sandage, M., \& Kristian, J. 1975, Chicago (University of Chicago Press), Stars and Stellar Systems, 9, 839

Sodroski, T. J., Odegard, N., Arendt, R. G., et al. 1997, ApJ, 480, 173

Strong, A. W., Bloemen, J. B. G. M., Dame, T. M., et al. 1988, A\&A, 207,1

Theis, Ch., \& Kohle, S. 2001, A\&A, 370, 365

Thronson, H. A., Hunter, D. A., Telesco, C. M., et al. 1987, ApJ, 317, 180 